\title{
The Glance software package
}

\author{
SUNIL SUBRAO PAI and FRANCIS T. DURSO \\ Texas Tech University, Lubbock, Texas
}

\begin{abstract}
We describe the Glance software package, which takes advantage of tablet PC technology to control experiments that require participants to reconstruct complex stimuli, as with the Chase and Simon (1973) chess reconstruction task, and to present tests that require participants to refer briefly to critical displays, as with the digit-symbol task. Participants press the space bar, which allows access to the stimulus and, simultaneously, prohibits drawing on the tablet. At release of the space bar-or optionally, after some period of time - the stimulus is hidden, and the drawing tools are enabled. Output from Glance allows analysis as fine grained as each stroke or as coarse as the entire image.
\end{abstract}

There are a variety of experimental paradigms in which a participant is presented with a display for a limited amount of time. In many of these paradigms, the behavior observed is a simple buttonpush, and the dependent variables are response times and error rates. However, the outputs can be more complicated. For example, a collection of unrelated items might be dumped from a purse, and the participant given a limited time to look at the collection before reproducing it. A more complicated version of such a paradigm is the classic Chase and Simon (1973) paradigm: Chess players glanced at active chess positions and were asked to reproduce those positions on an unoccupied board. The active position was on one side of a divider, and the empty board on the other. The chess player glanced around the divider at the active board and then back at the unoccupied board to reproduce the position. The participant would continue this glance-reconstruct sequence until the position had been reproduced. Researchers interested in the details of such tasks often use video recordings, in order to determine the number of glances, the timing of the glances, and so on. This paradigm has become a classic in the cognitive literature and has been used in various contexts over the past 30 years, continuing up to today (e.g., Gobet \& Clarkson, 2004; Gobet \& Simon, 1996). Currently, for example, we are engaged in applying a Chase and Simon type methodology to reconstruction of complex information displays. The Federal Aviation Administration is interested in developing a metric for assessing the information complexity in air traffic control displays. It was for this experimental purpose that the Glance software was developed. Although programs exist

Glance was developed in VisualBasic 6.0, using the TabletPC Platform Software Development Kit, Version 1.5. The program and the source code are available for download at www.depts.ttu.edu/psychology/cogergolab. We thank Rebekah Smith for the discussion that led to Glance. Thanks also to the FAA's Information Complexity Team and to Aaron Perez for his help. Correspondence should be addressed to either S. S Pai, 12308 Feather Wood Dr. \#42, Silver Spring, MD 20904 (e-mail: sun_pai@ yahoo.com) or F. T. Durso, Department of Psychology, Texas Tech University, P.O. Box 42051, Lubbock, TX 79409 (e-mail: frank.durso@ttu .edu). that allow specific reproduction, such as moving a virtual chess piece with a mouse, to our knowledge software with the features of Glance is not publicly available.

In addition to experimental procedures, there are assessment tools that have a similar flavor. For example, the digit-symbol substitution task Wechsler Adult Intelligence Scale (Wechsler, 1955) of the requires the examinee to view pairings of digits and symbols and then to reproduce the appropriate digit when given a symbol (see, e.g., Bryan \& Luszcz, 1996). The examinee must glance to the table of pairings whenever a symbol is presented that does not bring to mind the appropriate digit. Similarly, the Group Embedded Figures Test (Oltman, Raskin, \& Witkin, 1971) requires participants to glance at target-letter pairings whenever they do not recall which camouflaged target figure they are to find.

We have developed the Glance software to facilitate such studies and examinations while providing the type of enhanced data collection and presentation capabilities that digital technology allows. The program runs on all tablet PCs with Windows XP Tablet PC edition. For nontablet PCs, it runs on all versions of Windows with tablet PC components - that is, with ink control installed and a pointing device (the tablet PC components are downloadable from www.microsoft.com/downloads). The software has two executables, one to collect data and one to view output.

Figure 1 shows a typical study in which Glance is used. A stimulus is presented to the participant and remains visible as long as the space bar is depressed. When the space bar is released, the participant reconstructs on a digital tablet as much of the display as he or she remembers. The participant then presses the space bar again, and the inspect-reconstruct cycle continues until the display is reproduced. Glance provides options for controlling the displays of the target stimulus, the form (or background) on which the reconstruction takes place, the temporal parameters of the display, and the sampling of the output image storage.

Figure 2 shows the Glance program in the middle of a trial in which the participant is trying to reconstruct a stimulus (A) on the digital tablet (B). In the illustrative 

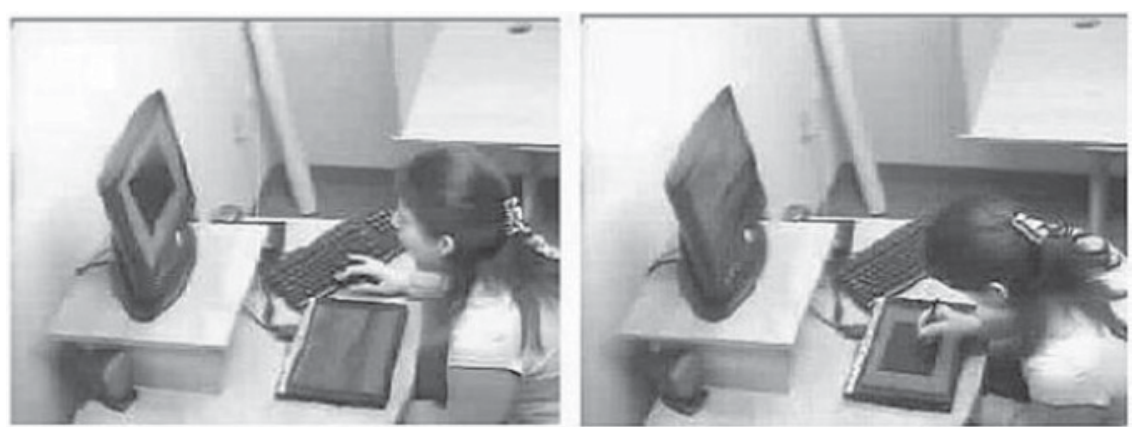

Figure 1. Picture of a participant using Glance.

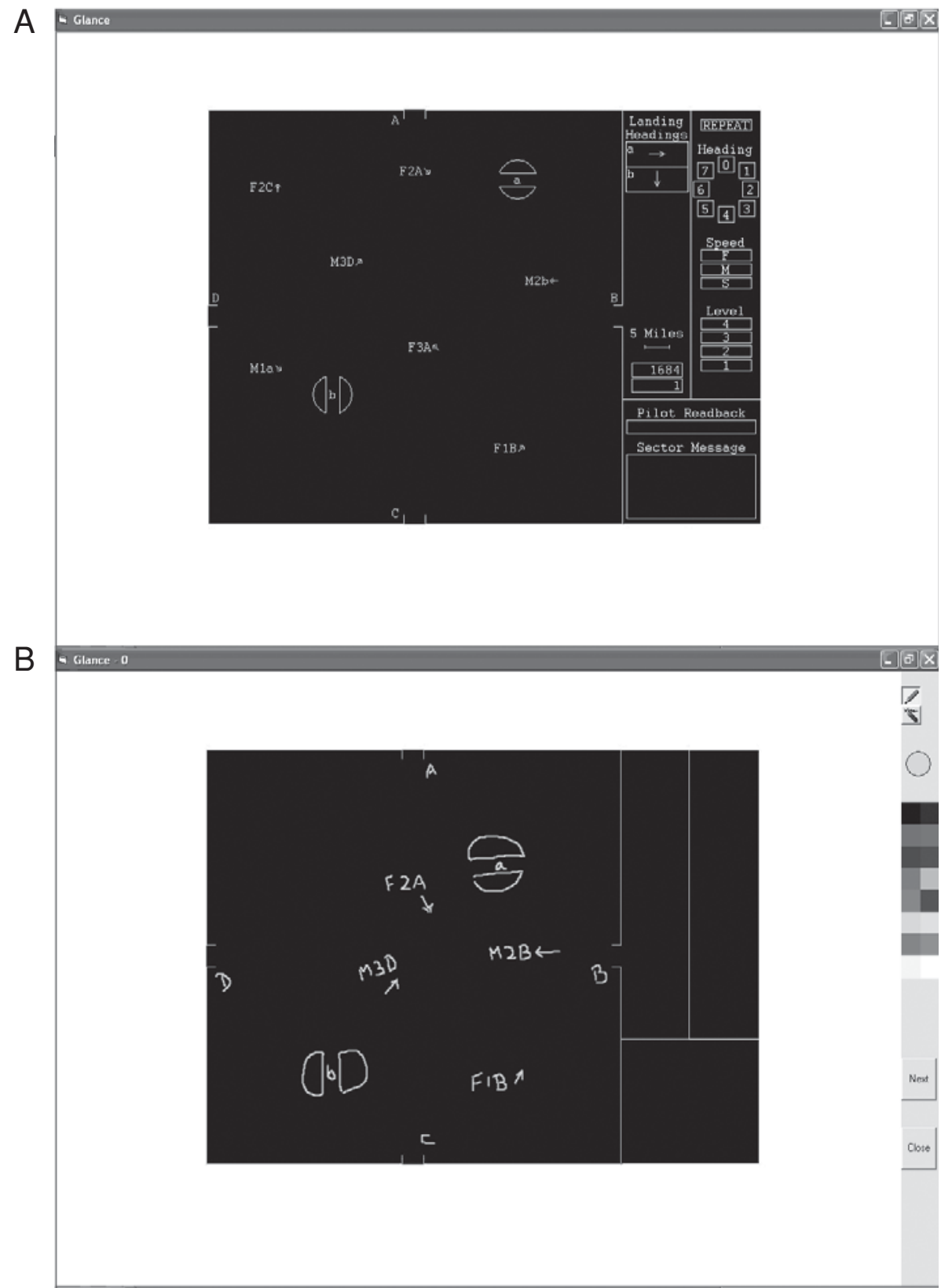

Figure 2. A stimulus (A) reproduced (B) using the Glance software. 
figure, the stimulus is a snapshot of a low-fidelity air traffic control display. The participant holds down the space bar and looks at the display. When the space bar is released, the stimulus disappears, and the participant uses the drawing tools to reconstruct another part of the display. In this example, the participant was given a background (i.e., form) on the tablet's drawing surface containing lines defining gates in the airspace and lines dividing the operational buttons. In the figure, the participant has reproduced the two airports, labeled the gates, and sketched four of the aircraft.

\section{Conducting Experiments}

The stimulus and the form files needed for the experiment can be created, scanned, or otherwise generated using any image-editing software, such as MS Paint or Adobe Photoshop. The files must be in BMP format and named stim0.bmp, stim1.bmp, . . stim N.bmp for stimulus files and form0.bmp, form 1.bmp, . . form N.bmp for form files. The stimulus and form files must be present in the "Images" subfolder under the main folder that contains the programs. The maximum number of stimulus or form files that the program can handle is 100 .

Initiating the Glance program brings up a dialog box that has been carefully designed to allow the experimenter to customize the experiment. The program provides several options for conducting the experiment, such as displaying the stimulus in a sequential order or randomizing it, with or without a background form. The experimenter can link a form for each stimulus or can even have a common form for the entire experiment. The results can be saved to allow analysis as fine-grained as each stroke or as coarse as the entire image at each glance. The number of glances to the stimuli for a participant can be controlled, as well as the time allowed for each glance, or can be left to the participant's discretion. These features make the program useful for a wide variety of procedures, allowing the experimenter greater control over the details of the experiment.

The illustrative dialog box (see Figure 3) displays the options selected on the air traffic control experiment. The number of stimuli used here is 25 , with one common background form. The stimulus will be displayed as long as the space bar is pressed; trials occur in sequential order. The maximum number of glances allowed for each trial is 50, and the image for analysis will be saved at each glance.

Clicking OK will bring up the drawing area, where the participant can write or draw. The participant must press the space bar to view the stimulus. The stimulus will be hidden either after a fixed interval or when the space bar is released. The participant can then try to reconstruct the stimulus on the drawing area, using the tools provided on the right-hand side of the tablet's window. Drawing functions are disabled when the stimulus is visible. Clicking the Next button will move the experiment on to the next trial.

As another illustration of Glance, consider implementation of the digit-symbol task. There would be one stimulus stored in the stim0.bmp file. The file would contain an image of the initial digit-symbol pairing table (Figure 4, top), which the participants will consult, if necessary, during the substitution task. The number of forms (Figure 4, bottom) would reflect the number of trials desired. Selecting the save-each-stroke option would save images at each stroke, which should correspond to a digit.

\section{Output}

Glance has an output program (see Figure 5) that allows the researchers to study the reconstruction process, including the timing and order of the subimages created. The output program helps in viewing the sequence of images saved for analysis. A particular trial can be accessed by opening any glc file in the trial directory. The images can be viewed in the order in which they were saved, thus replaying the entire experiment, providing all the vital information needed for analysis. The experimenter can move back and forth among these images, using the next and the back buttons.

The view_time.txt file in the trial directory contains the time when the participant viewed the stimulus. The start time is the time the participant started viewing the stimulus, and the stop time is the time the participant stopped

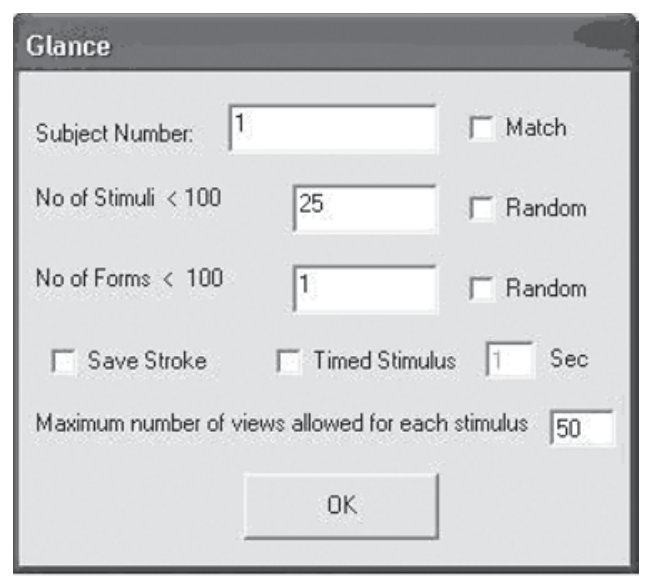

Figure 3. Input options for Glance. 
viewing the stimulus. The view_time.txt file may look like this:

\section{Start: 2.2 Stop: 5.2,}

Start: 11.4 Stop: 13.4

Start: 21.2 Stop: 25.5 ,

and so on. This indicates that the participant was viewing the stimulus for the time between 2.2 and $5.2 \mathrm{sec}$, then between 11.4 and $13.4 \mathrm{sec}$, and finally between 21.2 and $25.5 \mathrm{sec}$. The timings are noted with $0.1-\mathrm{sec}$ accuracy. The program cannot be used as is when more timing precision is required; precision can be increased up to $1 \mathrm{msec}$ by making the simple changes in the source and running the program on machines with higher processing speeds and RAM. The program uses the VisualBasic Timer control; more information on its accuracy can be found at msdn .microsoft.com/library/default.asp?url=/library/en-us/ vbcon $98 / \mathrm{html} / \mathrm{vbcontimercontrol.asp.}$

The output program needs the "Images" folder with the form files in it to run. The files saved are in ink-serialized format and can be viewed only through the output program. We have yet to solve the incompatibility problem that prevents saving the ink-serialized images to some standard format.

\section{Conclusion}

In our work, we have used Glance primarily to control experiments that require the reconstruction of air traffic control displays. The portability of today's tablet PCs and flat screen monitors gives us the potential to test our participants at sites convenient for them. Previously, obtain-

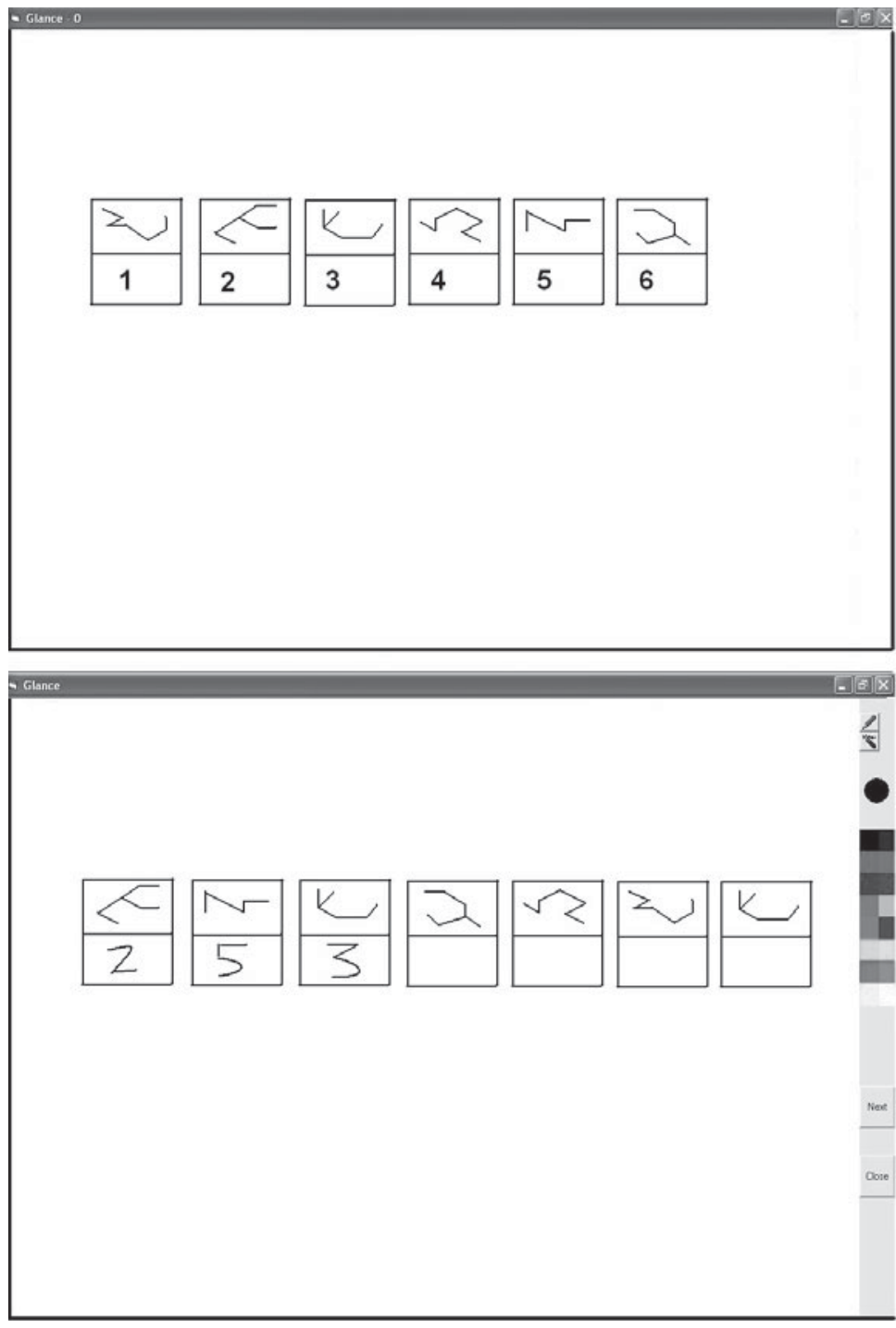

Figure 4. Screen shots of Glance during the digit-symbol task. The basic digit-symbol pairing (top) is available for viewing by pressing the space bar. One of several potential forms is partially completed (bottom). 


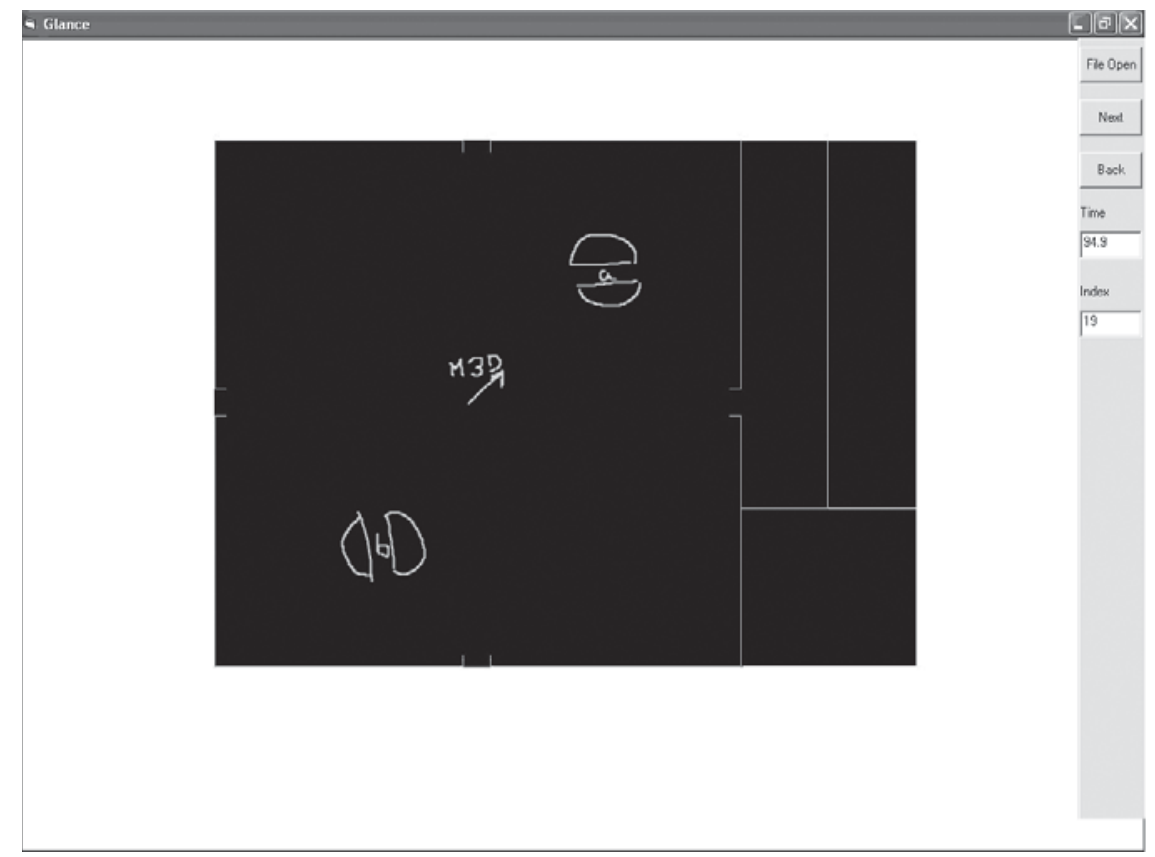

Figure 5. Output program showing the image after the 19th stroke, 94.9 sec after the trial started. Note the timing information presented.

ing this type of data from, for example, expert air traffic controllers required either transporting video cameras and other equipment or having busy professionals travel to the lab. In addition, the data captured by Glance help alleviate some of the problems associated with analyzing video tapes.

\section{REFERENCES}

Bryan, J., \& LuszcZ, M. A. (1996). Speed of information processing as a mediator between age and free-recall performance. Psychology \& Aging, 11, 3-9.
Chase, W. G., \& Simon, H. A. (1973). Perception in chess. Cognitive Psychology, 4, 55-81.

Gobet, F., \& Clarkson, G. (2004). Chunks in expert memory: Evidence for the magical number four . . . or is it two? Memory, 12, 732-747.

Gobet, F., \& Simon, H. A. (1996). Recall of random and distorted chess positions: Implications for the theory of expertise. Memory \& Cognition, 24, 493-503.

Oltman, P. K., Raskin, E., \& Witkin, H. A. (1971). Group Embedded Figures Test. Palo Alto, CA: Consulting Psychologists Press.

Wechsler, D. (1955). WAIS: Wechsler Adult Intelligence Scale. New York: Psychological Corporation.

(Manuscript received May 29, 2004;

revision accepted for publication February 7, 2005.) 